\title{
La Simulación Clínica en Atención Primaria de Salud en contexto de docencia: una experiencia con estudiantes de Fonoaudiología
}

\author{
Clinical Simulation in Primary Health Care in the \\ context of teaching: an experience with Language \\ and Hearing undergraduates
}

\author{
Mario Bustos \\ Departamento de Fonoaudiología \\ Universidad de Chile \\ Claudia Arancibia \\ Departamento de Fonoaudiología \\ Universidad de Chile \\ Nelson Muñoz \\ Departamento de Fonoaudiología \\ Universidad de Chile \\ Josefina Azócar \\ Departamento de Fonoaudiología \\ Universidad de Chile
}

\section{Contacto con el autor: Mario Bustos Santiago - Chile Correo-e: mbustosr@uchile.cl}

Recibido: 07/12/2017 Aceptado: 19/06/2018

\begin{abstract}
RESUMEN
El objetivo del presente trabajo fue describir y comparar el desempeño de estudiantes de Fonoaudiología en dos actividades de ECOE (Evaluación Clínica Objetiva Estructurada) de la asignatura "Integrado Clínico en Salud Primaria" de la carrera de Fonoaudiología de la Universidad de Chile. Para ello se realizó un estudio no experimental y transversal que consideró una muestra total de 29 estudiantes de IV año. Se analizaron 2 actividades de simulación clínica a un mismo grupo de estudiantes, la primera con evaluación de tipo formativa y la segunda con evaluación de carácter sumativa. Ambas evaluaciones contaron con tres instancias de desempeño: atención individual de usuarios, completación de ficha clínica y rendimiento en reunión con un equipo de salud. Los resultados mostraron un buen rendimiento de los estudiantes en la evaluación de las diferentes habilidades de simulación clínica no técnica en atención primaria en salud. Además, se observó un mayor rendimiento en la instancia de evaluación sumativa (segunda evaluación) comparada con la formativa en las tres instancias de desempeño medidas. Estas diferencias fueron estadísticamente significativas $(p<0,05)$. Estos resultados permiten concluir que las actividades realizadas facilitaron el logro de las competencias esperadas en la asignatura. La simulación como metodología favoreció la formación del programa, posicionando a esta actividad como una herramienta útil de aprendizaje a estudiantes previo a la atención de usuarios o interacción con grupos.
\end{abstract}

Palabras clave: paciente simulado, competencia clínica, fonoaudiología, atención primaria de salud.

\begin{abstract}
The main goal of this study was to describe and compare the performance of undergraduate students of speech language therapy in the activities of OSCE (Objective Structured Clinical Evaluation) of the subject "Integrated Clinical in Primary Health" of the career of Speech, Language and Hearing Sciences of the University of Chile. A non-experimental, crosssectional study was conducted with a total sample of 29 fourth-year students. Two clinical simulation activities were analyzed for the same group of students, the first one was a formative evaluation and the second one was a summative evaluation. Both activities inspect three kinds of performance; individual attention of users, completion of clinical record, and performance in meeting with a health team. Results showed that students performed well on the evaluation of different non-technical clinical simulation skills in primary health care. Performance was higher on summative evaluation (second evaluation) as compared with the summative one in the three types of observed evaluations. These differences were statistically significant $(p<0.05)$. Results show that the activities helped students achieve the expected competences. The simulation as a methodology favored the formation of the program, positioning this activity as a learning tool, an initiative of students to the attention of users or interaction with groups.
\end{abstract}

Keywords: patient simulation, clinical competence, speech, language and hearing sciences, primary health care. 


\section{Introducción}

Desde el año 2006, la Escuela de Fonoaudiología de la Universidad de Chile inicia un proceso de innovación curricular en el marco de la modernización del pregrado, establecido en el Plan de Desarrollo de la Universidad y la Facultad de Medicina. Esta transformación tiene como propósito actualizar los planes de estudio para formar profesionales con alto sentido público, crítico y aptitudes que les permitan anticipar, impulsar y liderar procesos de cambios en los diversos órdenes de la vida social (Universidad de Chile, 2011).

La innovación curricular implica actualizar metodologías de enseñanza-aprendizaje acorde a los cambios en los paradigmas de formación profesional universitaria que posicionan al estudiante como un agente activo de su formación y a los docentes como facilitadores del proceso educativo (McLean \& Gibbs, 2010). Para lograr este objetivo, se implementó un currículum basado en competencias, en el cual el estudiante debe actuar de manera pertinente en un contexto particular, siendo capaces de dar razón de las decisiones adoptadas, haciéndose responsables de las mismas y sus efectos (Universidad de Chile, 2011).

En este nuevo plan que entró en vigencia el año 2013, la Escuela de Fonoaudiología elaboró un análisis de necesidades formativas y ámbitos de desempeño, con la generación de diferentes dominios profesionales. En esta dirección, se propone una ampliación en los contextos de participación del fonoaudiólogo hacia el entorno social y familiar de una persona o comunidad, que requiera de apoyo profesional (Indicador de logro 2.4 .6 de la Subcompetencia 2 del dominio de intervención). Esta nueva línea de formación emergente busca incorporar temáticas, ámbitos de intervención y campos de acción en concordancia con el desarrollo disciplinar actual, las necesidades sanitarias del país y las políticas públicas (Arancibia, Coloma, \& Peñaloza, 2015; Escuela de Fonoaudiología, 2013). Es así como al consultar las políticas ministeriales del sector salud, es posible distinguir en la Reforma del sector salud la implementación de un Modelo de Atención Integral en Salud con enfoque familiar y comunitario a lo largo del país, en el cual la Atención Primaria en Salud tiene un rol fundamental. Esto, ya que se le considera una plataforma para llevar los servicios lo más cerca posible de las personas, mejorar la calidad de las prestaciones, completar la instalación de los equipos de salud, abrir nuevos espacios a la participación ciudadana y establecer la promoción de la salud en las políticas locales (Ministerio de Salud, 2008, 2013).

En este escenario, es necesario que los futuros profesionales comprendan el Modelo de Salud Familiar y cuenten con las competencias necesarias para su inserción en los programas de salud primaria vigentes. Con esta propuesta, se pretende pasar de un modelo biomédico a un modelo biopsicosocial, con una visión integral de la situación de salud de individuos, familias y comunidades (Organización Panamericana de la Salud \& Organización Mundial de la Salud, 2011). Acogiendo los requerimientos del Ministerio de Salud (MINSAL), la Escuela de Fonoaudiología de la Universidad de Chile propone la realización de la asignatura "Integrado Clínico en Salud Primaria", la que tiene como propósito formativo que el/la estudiante comprenda y reflexione respecto del rol del fonoaudiólogo en el contexto de atención primaria, teniendo en cuenta el 
modelo de salud familiar, orientado a trabajar por el bienestar de la persona, familia y comunidad a través de un abordaje integral, oportuno, de alta calidad y resolutivo (Ministerio de Salud, 2008). Su aporte al perfil de egreso contribuye a las competencias en la evaluación, diagnóstico y tratamiento de personas en centros de salud primaria, aportando a la calidad de vida de personas.

Una de las estrategias de enseñanza de aprendizaje en esta nueva asignatura es la Simulación Clínica en salud, modalidad que tiene evidencia e impacto sobre el aprendizaje disciplinar en la formación de estudiantes (Corvetto et al., 2013). Por lo anterior, el objetivo del presente artículo es describir y comparar el desempeño de estudiantes en dos actividades de ECOE (Evaluación Clínica Objetiva Estructurada) de la asignatura "Integrado Clínico en Salud Primaria" de la Carrera de Fonoaudiología de la Universidad de Chile.

\section{Simulación Clínica}

En la actualidad se ha incorporado la simulación clínica en el currículum de ciencias de la salud, debido a que múltiples estudios han evidenciado su validez y utilidad como estrategia didáctica en la formación de competencias en estudiantes de pregrado y postgrado (Escudero, Fuentes, Gonzalez, \& Corvetto, 2017). Esta modalidad permite a los estudiantes la repetición sistematizada de los procesos físicos y mentales necesarios para el desarrollo de las competencias y lo que es más importante, permite aprender de la equivocación en ese ambiente de aprendizaje (Amaya, 2010).

La simulación clínica toma un lugar importante a la hora de realizar innovaciones curriculares, al desarrollar competencias que no se logran a través de las clases expositivas o aprender del error con usuarios reales.

Las llamadas competencias de los profesionales de la salud se han definido como la práctica clínica basada en el empleo juicioso del conocimiento y del razonamiento clínico, junto con habilidades técnicas y de comunicación, sin dejar de lado aspectos valóricos y actitudinales; es así como, el ser competente implica una acción que beneficia a los usuarios y a la comunidad a la que pertenecen (Vásquez-Mata G. 2009). Una vez que el estudiante ha adquirido conocimiento y habilidades de comunicación, es posible complejizar el desarrollo de estas competencias mediante el uso de la simulación clínica. Esta metodología supone recrear situaciones clínicas en un ambiente controlado, seguro y estandarizado (Díaz, Arancibia, \& Bozzo, 2013).

La simulación clínica se ha convertido en un instrumento de gran utilidad para capacitar a los estudiantes a través de un entrenamiento sistematizado de situaciones que puede encontrar frecuentemente en el momento de enfrentar la realidad del paciente (Amaya, 2010).

Esta actividad entrega ventajas frente a otras metodologías de aprendizaje en el proceso de formación en competencias. El desempeño en un contexto protegido, supervisión continua, enfrentamiento a situaciones profesionales estimulantes, repetición de procedimientos para la adquisición de habilidades, entrenamiento de aspectos clínicos que en condiciones normales pueden requerir meses o años, transferencia de habilidades adquiridas a la realidad, aumento de seguridad a los pacientes disminuyendo los errores 
clínicos pueden ser considerados como ventajas (McGaghie, Issenberg, Petrusa, \& Scalese, 2010).

Por último, es importante señalar que la simulación clínica se adapta a las necesidades de aprendizaje del estudiante y resulta ser una manera eficaz de entrenar actitudes o habilidades no técnicas, como son el trabajo en equipo, la comunicación, la toma de decisiones, el liderazgo o el pensamiento crítico entre otros (Farrés Tarafa et al., 2015).

\section{Simulación Clínica y Atención Primaria en Salud}

La Organización Panamericana de la Salud (OPS) ha tomado la iniciativa continental en el campo de las competencias para el sector salud, particularmente en torno a la Atención Primaria de Salud (APS). A partir del 2005, sitúa en sus documentos este tema como de importancia crítica, para que los estados miembros se aboquen al tema de la gestión del recurso humano para la APS a partir de un modelo de competencias (Macinko, Montenegro, Nebot Adell, Etienne, \& Grupo de Trabajo de Atención Primaria de Salud de la Organización Panamericana de la Salud, 2007; Organización Panamericana de la Salud, 2008).

Existen experiencias de Escuelas de Medicina a nivel Iberoamericano que han considerado el desarrollo de competencias en la APS a nivel de pregrado mediante el uso de la Simulación Clínica. Según un estudio elaborado por Vigo Cuza et al. (2015), se logra evidenciar que los profesores que utilizan la simulación clínica la valoran como un recurso para el proceso enseñanza aprendizaje en los escenarios de formación de la APS, pues logra establecer una relación entre el saber, hacer y ser del profesional de salud con el beneficio final en el usuario y su entorno.
En otro contexto, en el año 2009, la Sociedad Española de Pediatría de Atención Primaria (SEPEAP) diseñó y desarrolló cursos de Simulación Clínica centrado en las necesidades de aprendizaje y capacitación de sus miembros, con buenos resultados a nivel de percepción, pues sus estudiantes valoran este tipo de formación al considerarla pertinente y muy útil para su actividad laboral (Sánchez Santos et al., 2010).

En Chile, la implementación del modelo de atención integral con enfoque familiar y comunitario requiere de profesionales con competencias específicas y complementarias, que permitan cubrir la cartera de servicios orientada a satisfacer las necesidades de salud integral de la población a cargo. Se pretende alinear las exigencias del modelo con el perfil del recurso humano (Ministerio de Salud, 2013). De acuerdo a Montero et al. (2009), las competencias profesionales necesarias para un óptimo desempeño en la APS serían el conocimiento técnico para resolver problemas específicos del área, conocimientos de herramientas de intervención familiar, fundamentos de la promoción en salud, cambios conductuales, educación participativa, comunicación efectiva, trabajo en equipo, fijación de metas personales, preocupación por exactitud, iniciativa, eficiencia, flexibilidad, creatividad y enfoque al usuario, características que podrían ser desarrolladas y evaluadas mediante la Simulación Clínica.

Durante la asignatura abordada, se propone la ejecución de un ECOE (Evaluación Clínica Objetiva Estructurada) que consiste en un circuito de estaciones secuenciales en el que se utilizan pacientes simulados estandarizados o casos (Corvetto et al., 2013). En el programa se propone como una 
metodología innovadora para el desarrollo de competencias necesarias para el trabajo en APS. Es así como esta actividad evalúa las habilidades no técnicas que el estudiante pone de manifiesto para resolver problemas, la comunicación efectiva para entrega de información, trabajo en equipo y flexibilidad para abordar las situaciones propuestas (Hagemann et al., 2017). Lo anterior contribuye a la competencia de intervención: "Dominar el proceso de intervención fonoaudiológica, considerando a la persona que requiere apoyo fonoaudiológico y su contexto (social y cultural), sobre la base de fundamentos disciplinares y ético" y a la competencia de compromiso social: "Actuar desde su rol como fonoaudiólogo para contribuir al bienestar de las comunidades, especialmente en aquellas más vulnerables".

El aporte de este curso al perfil de egreso está relacionado con las competencias en evaluación, diagnóstico y tratamiento de personas en los centros de salud primaria, aportando a la calidad de vida de personas y el desarrollo de la disciplina en este tipo de centros de atención.

\section{Metodología}

\section{Tipo de Estudio}

Estudio de tipo no experimental y transversal que consideró una muestra total de 29 estudiantes de IV año de la carrera de Fonoaudiología de la Universidad de Chile año 2016.

\section{Caracterización de la muestra}

La muestra estuvo constituida por 28 mujeres y 1 hombre entre 20 y 23 años, que cumplían requisitos curriculares para inscribir el curso Integrado Clínico en Salud Primaria, asignatura obligatoria impartida el VII Semestre de la carrera.

\section{Propósito de la actividad y su evaluación}

El propósito de la actividad simulada fue aportar a los siguientes resultados de aprendizaje declarados en la asignatura:

1. Analizar el sistema de atención primaria de salud chileno, para visibilizar el rol del fonoaudiólogo y su quehacer dentro de ese nivel.

2. Colaborar en equipos de salud de distintos dispositivos de atención primaria donde esté inserto un fonoaudiólogo.

Tanto la evaluación formativa como la sumativa buscaban evaluar ambos propósitos con calificaciones de promediación simple de 3 calificaciones resultantes:

1. Calificación de ficha electrónica de evaluación: evaluación realizada por un académico.

2. Calificación por atención al usuario: evaluación realizada por un actor en cada estación de atención individual.

3. Calificación por desempeño grupal en reunión: evaluación realizada por un académico con entrega adicional de retroalimentación cualitativa sobre colaboración en equipo.

\section{Descripción de la actividad simulada}

Se realizaron 2 actividades de simulación a un mismo grupo de estudiantes. La primera evaluación se realizó en abril de 2016 y fue de tipo formativa. La segunda, de carácter sumativo, se realizó en agosto de 2016. Se utilizaron para la actividad dependencias 
del Centro de Habilidades Clínicas de la Universidad de Chile ( $\mathrm{CHC})$.

La tabla 1 presenta un desglose de los dos tipos de actividades realizadas.

Tabla 1

Distribución de estaciones de simulación.

\begin{tabular}{lccc}
\hline Espacio & Estudiantes & Actores & $\begin{array}{c}\text { Tiempo } \\
(\mathrm{min})\end{array}$ \\
\hline $\begin{array}{l}\text { Box } \\
\text { Sala } \\
\text { Reunión }\end{array}$ & 1 & 1 & $\begin{array}{c}30(15 \text { por } \\
\text { usuario })\end{array}$ \\
\hline
\end{tabular}

Para el examen se dispuso de seis espacios habilitados: cinco boxes y una sala de reuniones. En cada box, los estudiantes debían atender a dos actores que simulaban el rol de usuario. La tarea de los estudiantes era orientar con habilidades de intervención no técnica a cada usuario, ayudándolos a partir del motivo de consulta y antecedentes de cada uno. En la sala de reuniones, 6 estudiantes debían interactuar con un actor que interpretaba a un director de un CESFAM. De los 30 minutos de duración de la actividad, 22 estaban dedicados a la preparación de argumentos y defensa de un proyecto frente al director y 8 minutos restantes consistieron en retroalimentación del académico evaluador de esa instancia, a partir del desempeño de los estudiantes en grupo se obtuvo una nota individual.

\section{Estaciones con usuarios simulados de atención individual}

Los actores fueron entrenados por el equipo encargado de la realización de las evaluaciones estructuradas, a fin de estandarizar sus roles. Las estaciones fueron evaluadas mediante 2 de 3 casos posibles (Tabla 2); la primera mediante la evaluación de las fichas electrónicas y la segunda realizada por cada usuario simulado luego de ser atendido por cada estudiante. El desempeño de los alumnos se registró en pautas de evaluación digital previamente diseñadas, en donde los académicos y actores podían revisar los objetivos y habilidades esperadas para cada estudiante. Cada pauta contaba con su propio modo de utilización; en el caso de las fichas electrónicas, estas tenían una pauta de respuestas esperadas con puntaje adjudicado a cada respuesta correcta, mientras que los instrumentos de evaluación usados por los actores contaban con opciones de logro de cada objetivo con puntajes. Los contenidos tratados en cada estación fueron previamente presentados a los estudiantes en la asignatura. 
Tabla 2

Especificaciones de los usuarios simulados para atención de estudiantes en box.

\begin{tabular}{|c|c|c|}
\hline Caso & Descripción del caso & Objetivos \\
\hline Usuario 1 & $\begin{array}{l}\text { Asiste al centro de salud primaria de referencia } \\
\text { madre de niño de } 2 \text { años, luego que la enfermera } \\
\text { que hizo el control sano realizara una derivación al } \\
\text { programa Chile Crece contigo para atención, ya } \\
\text { que el menor nació prematuro y presenta un } \\
\text { retraso de lenguaje evidente. }\end{array}$ & $\begin{array}{l}\text { Se evaluó la entrega correcta de orientaciones en } \\
\text { torno al programa, la capacidad de responder a } \\
\text { inquietudes de la madre, el razonamiento del } \\
\text { estudiante en torno al caso y las correcta } \\
\text { completación de la ficha. }\end{array}$ \\
\hline Usuario 2 & $\begin{array}{l}\text { Joven es llevado al centro de salud en una } \\
\text { oportunidad ya que ingresa al "Programa Atención } \\
\text { Domiciliaria Integral", debido a su condición de } \\
\text { salud y movilidad reducida luego de sufrir un } \\
\text { traumatismo luego de una pelea que lo dejo con } \\
\text { problemas motores y que lo ha mantenido } \\
\text { semipostrado desde hace } 1 \text { año sin poder seguir } \\
\text { estudiando su carrera universitaria y pasatiempos } \\
\text { de su vida diaria previa. El estudiante debe } \\
\text { notificar el inicio del programa, orientar y } \\
\text { contener al usuario, ya que aún no se encuentra } \\
\text { estable emocionalmente. }\end{array}$ & $\begin{array}{l}\text { Se evaluó la capacidad de entregar la información } \\
\text { con claridad, responder a las dudas del joven y } \\
\text { contenerlo si fuese necesario, además del llenado } \\
\text { correcto de la ficha electrónica. }\end{array}$ \\
\hline Usuario 3 & $\begin{array}{l}\text { Adulto mayor ingresa a programa tras ser } \\
\text { evaluada en EPAM anual y obtener la } \\
\text { caracterización de autovalente con riesgo. Es } \\
\text { derivada al box de Fonoaudiología para atención } \\
\text { semanal para trabajo cognitivo y entrenamiento } \\
\text { auditivo con su audífono, esto bajo las } \\
\text { consideraciones del programa. }\end{array}$ & $\begin{array}{l}\text { Se evaluó la capacidad de entregar la información } \\
\text { con claridad, responder a las dudas del joven y } \\
\text { contenerlo si fuese necesario, además del llenado } \\
\text { correcto de la ficha electrónica. }\end{array}$ \\
\hline
\end{tabular}

\section{Estación grupal de reunión de equipo de salud}

La sala de reuniones simulada contaba con un espejo unidireccional que permitía su visión desde una sala contigua. La actividad tenía por objetivo que los estudiantes demostraran sus habilidades comunicativas y argumentativas atingentes a los requerimientos de la dirección de un dispositivo de salud primaria. Para ello, se ambientó el lugar como una oficina de un CESFAM de una comuna rural y que contó con la participación de un director, quién debía recepcionar a 2 grupos de estudiantes, que argumentaban en favor de sus proyectos propuestos para el dispositivo de salud. En la tabla 3 se presentan las características del caso. 
Tabla 3

Caso estación reunión con equipo de salud.

\begin{tabular}{|c|c|c|}
\hline Caso & Descripción de la actividad & Objetivo \\
\hline $\begin{array}{l}\text { Reunión en Centro } \\
\text { de Salud Familiar }\end{array}$ & $\begin{array}{l}\text { Fonoaudiólogos fueron citados a una reunión } \\
\text { con la dirección del Departamento de Salud } \\
\text { Municipal de una comuna rural, en la cual se } \\
\text { pretende evaluar la factibilidad de la } \\
\text { incorporación de nuevos profesionales de salud } \\
\text { a la red de salud primaria de la comuna. El } \\
\text { grupo deberá argumentar la contratación de } \\
\text { nuevos profesionales bajo la necesidad de salud } \\
\text { de la comuna. El directorio del Depto. De Salud } \\
\text { tiene dudas sobre esta incorporación ya que la } \\
\text { división tiene otras necesidades. }\end{array}$ & $\begin{array}{l}\text { El objetivo buscó medir las habilidades de } \\
\text { organización del grupo, la síntesis de información } \\
\text { extraída de los datos de la comuna, los } \\
\text { argumentos, contrargumentos en el debate y las } \\
\text { habilidades pragmáticas de atingencia al } \\
\text { contexto. }\end{array}$ \\
\hline
\end{tabular}

\section{Análisis de Datos}

Los datos fueron analizados en el software $R(R$ Team, 2017). Debido a que el interés principal de este estudio era observar el cambio entre la medición formativa y la final, se decidió no comparar el rendimiento en las instancias de evaluación (ficha, box y reunión). Por tanto, a pesar de que el diseño permitía emplear una prueba de Anova mixto, se optó por realizar "Pruebas $t$ de muestras relacionadas" en cada una de las instancias comparando los dos momentos de medición. Los resultados se presentan a continuación.

\section{Resultados}

La presentación de los resultados considera dos instancias: la descripción de los descriptivos de las calificaciones finales obtenidas por los estudiantes en el ECOE, junto con la comparación del rendimiento entre los ECOE formativo y final.

\section{Calificaciones finales de cada estudiante}

Para la primera instancia de evaluación se describe el rendimiento de los 29 estudiantes en la instancia final de evaluación de habilidades con calificaciones en escala 1,0 a 7,0. En el Tabla 4 se observa el promedio obtenido por todo el grupo de estudiantes en las 3 instancias evaluadas, junto a la Desviación Estándar (DE) obtenida en cada actividad. La nota de aprobación para esta actividad fue de un 4,0 por lo que todos los estudiantes aprobaron la actividad.

Tabla 4

Promedio y DE en las 3 instancias finales evaluadas.

\begin{tabular}{lc}
\hline Instancia Evaluada & Promedio y DE \\
\hline Box & $6,18(0,67)$ \\
Ficha & $5,86(0,95)$ \\
Reunión & $5,9(0,96)$ \\
\hline
\end{tabular}

A continuación, se describen las calificaciones por cada estación de evaluación: 
a) Calificación final en ficha clínica en los boxes de atención ambulatoria:

En el gráfico 1, se observa que dos estudiantes obtuvieron una nota inferior a 4,0 en este ítem; un estudiante tuvo nota entre 4,0 y 4,9 ; cinco estudiantes rindieron entre 5,0 y 5,9 y veintiún estudiantes tuvieron nota entre 6,0 y 7,0. El promedio de notas obtenido en esta estación fue 5,8 con una desviación estándar de 0,955.

FICHA CLÍNICA

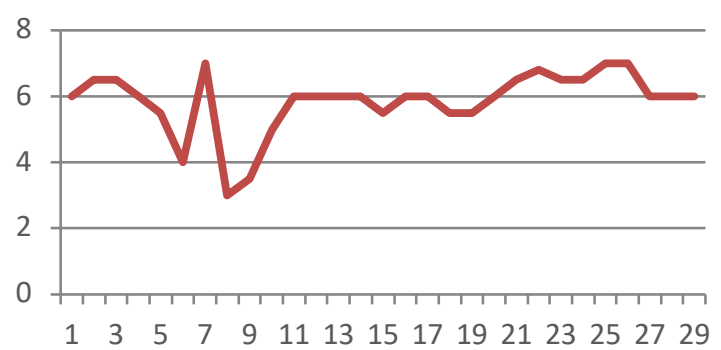

Gráfico 1. Rendimiento de cada estudiantes en ficha clínica.

\section{b) Calificación final de usuarios simulados en box de atención:}

Los datos que se presentan a continuación evidencian el rendimiento de los estudiantes en la estación de simulación de una atención ambulatoria, de acuerdo con la evaluación realizada por los actores que trabajaron en la simulación. En el gráfico 2 se observa que no hubo estudiantes con nota inferior a 4,0; un estudiante obtuvo nota entre 4,0 a 4,9; siete estudiantes entre 5,0 y 5,9 y veintiún estudiantes tuvieron nota entre 6,0 y 7,0. El promedio de notas obtenido en esta estación fue 6,2; con una desviación estándar de 0,662.

\section{ATENCIÓN BOX}

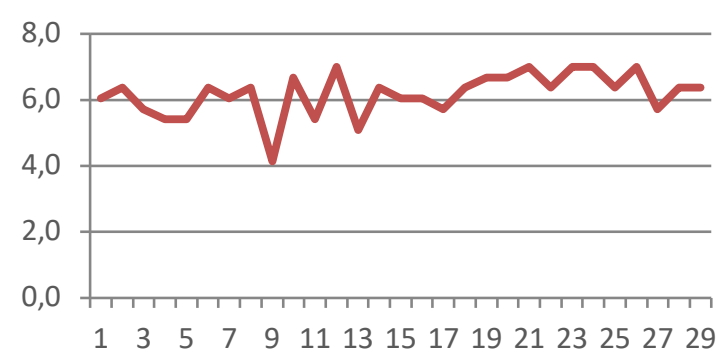

Gráfico 2. Rendimiento de cada estudiante en Box de atención ambulatoria.

\section{c) Calificación en estación de reunión equipo de salud:}

Los datos que se presentan a continuación evidencian el rendimiento de los estudiantes en la estación de simulación de una reunión clínica.

El gráfico 3 muestra que dos estudiantes obtuvieron nota inferior a 4,0 en este ítem; tres estudiantes obtuvieron una nota entre 4,0 y 4,9; nueve estudiantes tuvieron entre 5,0 y 5,9; y quince estudiantes tuvieron entre 6,0 y 7,0. El promedio de notas obtenido en esta estación fue 5,9; con una desviación estándar de 1.

\section{REUNIÓN EQUIPO DE SALUD}

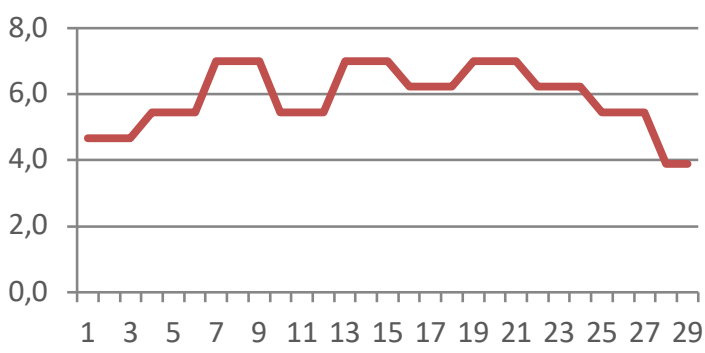

Gráfico 3. Rendimiento de cada estudiante en reunión equipó de salud. 


\section{Comparación entre los ECOE formativo y final}

Los resultados que se presentan a continuación se obtuvieron a partir de la comparación entre los rendimientos de los estudiantes en los ECOE formativo y final, expresado a través de la nota final de dichas instancias.

Tabla 5

Valores $p$ y d en las 3 instancias de evaluación.

\begin{tabular}{lcc}
\hline $\begin{array}{l}\text { Instancia de } \\
\text { evaluación }\end{array}$ & $p$ & $d$ \\
\hline Ficha & $<0,001$ & 0,88 \\
Box & 0,0274 & 0,53 \\
Reunión & $<0,001$ & 1,0 \\
\hline
\end{tabular}

En la tabla 5 se presentan los valores $P(p)$ obtenidas con pruebas $\mathrm{T}$ de muestras relacionadas (con hipótesis direccional) entre la calificación formativa y sumativa obtenida en las 3 instancias de evaluación. Los valores obtenidos evidencian una diferencia estadísticamente significativa en todas las instancias medidas, lo que revela que la nota promedio de los estudiantes en la evaluación formativa fue significativamente menor a la obtenida en la evaluación sumativa, en todas las instancias de evaluación $(p<0,05)$. Se entrega a la vez el Delta de Cohen (d) como medida de tamaño de efecto, donde se observa un cambio significativo en el cambio de las calificaciones obtenidas entre la primera y segunda oportunidad en todas las actividades medidas, con menor diferencia en la evaluación en la instancia "Box" (0,53).

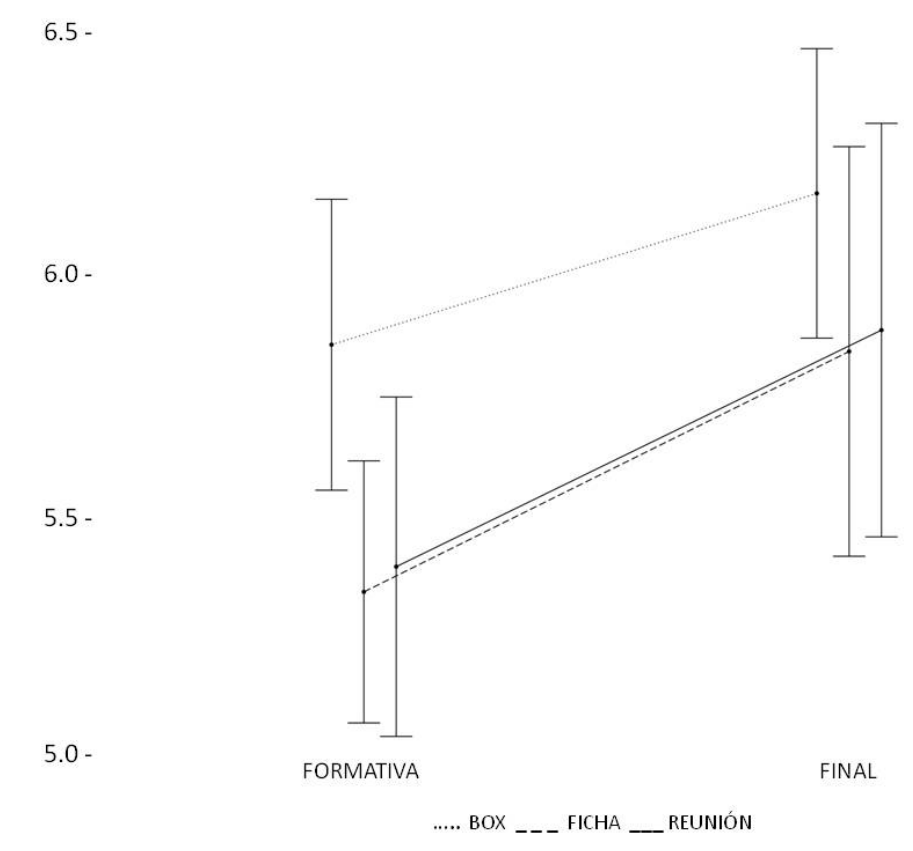

Gráfico 4. Promedio de las notas formativa y sumativa desglosadas por instancia de evaluación. 
El gráfico 4 de barras y líneas permite observar el cambio entre la primera y segunda medición. En el eje de las ordenadas se encuentra el rango de calificaciones de manera ascendente, donde se observa el aumento del rendimiento de los estudiantes en las diferentes instancias de evaluación. En el eje horizontal es posible observar el cambio de cada instancia evaluada, donde el comportamiento en la actividad "Ficha" y "Reunión" se muestra muy similar, mientras que la instancia "Box" se aleja un poco en relación a las notas obtenidas y a la magnitud del cambio logrado en la actividad sumativa.

\section{Discusión}

La formación universitaria bajo un modelo basado en competencias ofrece nuevas posibilidades $y$ desafíos para la evaluación de los aprendizajes esperados. En el caso de la asignatura de Integrado Clínico en Salud Primaria, la modalidad de simulación clínica bajo un modelo de ECOE buscó ser un instrumento posible en la medición de algunos aprendizajes esperados para el curso. Junto a lo anterior, la utilización de este tipo de evaluaciones podría estar contribuyendo a acercar a los estudiantes a contextos de situación práctica previo a la interacción real con usuarios de los servicios de salud (González \& Caballero, 2013).

Los resultados positivos de las calificaciones de los estudiantes en los diferentes escenarios propuestos podrían ser un aporte a la medición de los logros esperados en la asignatura. Cabe destacar que, si bien el instrumento de evaluación no fue validado previamente, este fue confeccionado por los académicos a cargo de la asignatura y por actores con experiencia en la evaluación de habilidades de simulación clínica no técnica. En ese sentido es relevante contar con instrumentos validados de evaluación, y al mismo tiempo, es necesario trabajar con simuladores capacitados en la entrega de retroalimentación y calificación a los estudiantes (Opazo Morales, Rojo, \& Maestre, 2017).

Con respecto a los desempeños en los diferentes casos de atención en box, el de menor rendimiento fue en el caso "Chile Crece Contigo", causado probablemente por la especificidad del caso planteado, debido a la cercanía de este contexto al que hacer fonoaudiológico. Por otro lado, la actividad de reunión de equipo de salud resultó ser una actividad probablemente innovadora y atingente para la formación en el modelo de salud primaria, donde también fueron considerados en su confección logros de aprendizaje esperados en el curso.

En relación con la comparación entre la evaluación formativa y sumativa de ECOE, este entrenamiento previo resultó ser un buen instrumento para mejorar el rendimiento de los estudiantes en todas las instancias propuestas de evaluación. La diferencia encontrada entre la evaluación "Box" en comparación a las otras 2 actividades, pudo deberse a que esta fue medida por actores calificados, quienes otorgaron notas más positivas a los estudiantes, algo que podría traducirse en que el cambio entre la evaluación formativa y sumativa fuera menor. Aun así, lo anterior se ajusta a que los procesos de evaluación en simulación clínica deberían contar siempre con actividades previas formativas, que presenten una estructura similar a lo evaluado formalmente, esto otorgaría mayor seguridad en los evaluados, reduciría los errores del proceso mejorando los tiempos y 
experiencia de los participantes (Vázquez-Mata \& Guillamet-Lloveras, 2009).

La sistematización y formalización de este tipo de ECOE posicionaría a esta actividad de simulación como una herramienta posible en la evaluación para la formación de estudiantes en salud primaria, aportando en parte a la formación clínica necesaria previo a que los estudiantes enfrenten situaciones reales de atención o interacción en salud (McGaghie et al., 2010). En este sentido este estudio es pionero en describir este tipo de actividades de formación en atención primaria del futuro profesional fonoaudiólogo, esto a nivel nacional como internacional. Lo anterior pone de manifiesto el seguir formalizando este tipo de instancia para contribuir contar con mejores herramientas para la docencia universitaria.

Si bien este formato de evaluación podría cubrir en parte la evaluación de las competencias propuestas en el curso, la simulación en salud es sólo un modelo de los muchos necesarios y existentes para el trabajo en atención primaria en salud. La formación en esta área necesita de mucha expertiz, variadas metodologías de aprendizaje y validación de los instrumentos de evaluación. Asimismo, es importante señalar que el éxito de este tipo de actividades requiere de infraestructura, recursos técnicos y humanos, los cuales son factibles de conseguir en el Centro de Habilidades Clínicas de la Facultad de Medicina de la Universidad de Chile.

La incorporación de la simulación clínica integrada al currículum podría ser una de las metodologías para desarrollar con mayor evidencia las competencias profesionales, de acuerdo al perfil de egreso declarado, por lo cual debería continuar siendo una estrategia relevante dentro del proceso de aprendizaje del profesional fonoaudiólogo. A su vez, la Universidad trabaja en ser pionera en el uso de la simulación clínica como un método formal de evaluación de habilidades, con espacios, personal y programas de formación destinados a esta área, todo lo cual corresponde a estándares internacionales en simulación clínica (Corvetto et al., 2013).

\section{Conclusión}

La realización del ECOE en el curso de Integrado Clínico en salud primaria facilitaría el logro de algunas competencias esperadas para el trabajo en atención primaria en salud. Por lo anterior, las actividades de simulación pueden resultar ser una metodología que favorece la formación de este ámbito de la salud en los estudiantes de fonoaudiología de la Universidad de Chile. A la vez, el uso de instancias formativas previas de evaluación favorecería a un mejor desempeño en la actividad simulada. Por lo anterior, la estructuración y entrenamiento guiado en este tipo de actividades, posicionaría este formato como una herramienta útil y positiva previa a la atención de usuarios o interacción de equipo en estos dispositivos de salud.

\section{Referencias}

Amaya, A. (2010). Simulación clínica: «aproximación pedagógica de la simulación clínica». Universitas Médica, 51(2), 204-211.

Arancibia, C., Coloma, C. J., \& Peñaloza, C. (2015). Análisis del proceso de innovación curricular en la Escuela de Fonoaudiología de la Universidad de Chile. Revista Chilena de Fonoaudiología, 14, 118-128. https://doi.org/10.5354/rcdf.v14i0.37696 
Corvetto, M., Bravo, M. P., Montaña, R., Utili, F., Escudero, E., Boza, C., ... Dagnino, J. (2013). Simulación en educación médica: una sinopsis. Revista médica de Chile, 141(1), 7079. https://doi.org/10.4067/S0034-98872013000100010

Díaz, M., Arancibia, C., \& Bozzo, S. (2013). Factibilidad de realizar un examen clínico objetivo y estructurado en la carrera de Fonoaudiología. Revista Hospital Clínico Universidad de Chile, 24, 123-129.

Escudero, E., Fuentes, C., Gonzalez, M., \& Corvetto, M. (2017). Simulación en educación para ciencias de la Salud: ¿Qué calidad hemos alcanzado en Chile? ARS MEDICA Revista de Ciencias Médicas, 41(3), 16-20. https://doi.org/10.11565/arsmed.v41i3.394

Escuela de Fonoaudiología. (2013). Plan de Formación (Documento Interno). Santiago: Facultad de Medicina, Universidad de Chile.

Farrés Tarafa, M., Miguel Ruiz, D., Almazor, A., Insa, E., Hurtado, B., Nebot, C., ... Roldán Merino, J. (2015). Simulación clínica en enfermería comunitaria. FEM: Revista de la Fundación Educación Médica, 18, s62-s66. https://doi.org/10.4321/S2014-98322015000300010

González, A. M., \& Caballero, A. R. (2013). Evaluación sumativa de los residentes mediante simulación: utilidad de los simuladores a escala real. FEM: Revista de la Fundación Educación Médica, 16(1), 41-47. https://doi.org/10.4321/S2014-98322013000100008

Hagemann, V., Herbstreit, F., Kehren, C., Chittamadathil, J., Wolfertz, S., Dirkmann, D., ... Peters, J. (2017). Does teaching non-technical skills to medical students improve those skills and simulated patient outcome? International Journal of Medical Education, 8, 101-113. https://doi.org/10.5116/ijme.58c1.9f0d

Macinko, J., Montenegro, H., Nebot Adell, C., Etienne, C., \& Grupo de Trabajo de Atención Primaria de Salud de la Organización Panamericana de la Salud. (2007). La renovación de la atención primaria de salud en las Américas. Revista Panamericana de Salud Pública, 21(2/3), 73-84. https://doi.org/10.1590/S1020-49892007000200003

McGaghie, W. C., Issenberg, S. B., Petrusa, E. R., \& Scalese, R. J. (2010). A critical review of simulation-based medical education research: 2003-2009. Medical Education, 44(1), 50-63. https://doi.org/10.1111/j.1365-2923.2009.03547.x
McLean, M., \& Gibbs, T. (2010). Twelve tips to designing and implementing a learner-centred curriculum: prevention is better than cure. Medical Teacher, 32(3), 225-230. https://doi.org/10.3109/01421591003621663

Ministerio de Salud. (2008). En el camino a Centro de Salud Familiar (p. 133). Santiago: Gobierno de Chile, Subsecretaría de Redes Asistenciales.

Ministerio de Salud. (2013). Modelo de atención integral con enfoque familiar y comunitario en establecimientos de la red de atención de salud (p. 197). Santiago: Gobierno de Chile, Subsecretaría de Redes Asistenciales.

Montero, J., Rojas, M. P., Castel, J., Muñoz, M., Brunner, A., Sáez, Y., \& Scharager, J. (2009). Competencias médicas requeridas para el buen desempeño en Centros de Salud Familiares en Chile. Revista médica de Chile, 137(12), 15611568. https://doi.org/10.4067/S0034-98872009001200003

Opazo Morales, E. I., Rojo, E., \& Maestre, J. M. (2017). Modalidades de formación de instructores en simulación clínica: el papel de una estancia o pasantía. Educación Médica, 18(1), 22-29. https://doi.org/10.1016/j.edumed.2016.07.008

Organización Panamericana de la Salud. (2008). Sistemas de Salud basados en la Atención Primaria de Salud - Estrategias para el desarrollo de los equipos de APS (p. 46). Washington: OPS.

Organización Panamericana de la Salud, \& Organización Mundial de la Salud. (2011). Implementación del modelo biopsicosocial para la atención de personas con discapacidad a nivel nacional (p. 33). Salvador: OPS / OMS.

Sánchez Santos, L., Rodríguez Núñez, A., Iglesias Vázquez, J. A., Civantos Fuentes, E., Couceiro Gianzo, J., Rodríguez Suárez, J., \& Fernández Sanmartín, M. (2010). Simulación avanzada para pediatras de atención primaria. Desarrollo de un programa itinerante y opinión de los participantes. Anales de Pediatría, 72(1), 55-61. https://doi.org/10.1016/j.anpedi.2009.08.017

Universidad de Chile. (2011). Modelo Educativo de la Universidad de Chile. Santiago: Ediciones Universidad de Chile. Recuperado de http://www.libros.uchile.cl/717

Vázquez-Mata, G., \& Guillamet-Lloveras, A. (2009). El entrenamiento basado en la simulación como innovación imprescindible en la formación médica. Educación Médica, 12(3), 149-155. 
Vigo Cuza, P., León Sánchez, B., Segrea González, J., López

Otero, T., Martínez López, H., \& León Sánchez, C. (2015). Simulación en la educación en el trabajo en los escenarios de formación de la Atención Primaria de Salud. MediSur, 13(2), 285-295. 